\title{
Blockchain in Oil and Gas Industry: Applications, Challenges, and Future Trends
}

This paper was downloaded from TechRxiv (https://www.techrxiv.org).

\section{LICENSE}

CC BY 4.0

SUBMISSION DATE / POSTED DATE

$18-10-2021 / 19-10-2021$

\section{CITATION}

Ahmad, Raja Wasim; Salah, Khaled; Jayaraman, Raja; Yaqoob, Ibrar; Omar, Mohammed (2021): Blockchain in Oil and Gas Industry: Applications, Challenges, and Future Trends. TechRxiv. Preprint. https://doi.org/10.36227/techrxiv.16825696.v1

$\mathrm{DOI}$ 


\title{
Blockchain in Oil and Gas Industry: Applications, Challenges, and Future Trends
}

\author{
Raja Wasim Ahmad, Khaled Salah, Raja Jayaraman, Ibrar Yaqoob, Mohammed Omar
}

\begin{abstract}
Today's systems, approaches, and technologies leveraged for managing oil and gas supply chain operations fall short in providing operational transparency, traceability, audit, security, and trusted data provenance features. Also, a large portion of the existing systems is centralized, manual, and highly disintegrated which make them vulnerable to manipulation and the single point of failure problem. In this survey, we explore the potential opportunities and applications of blockchain technology in managing the exploration, production, and supply chain and logistics operations in the oil and gas industry as it can offer traceability, immutability, transparency, and audit features in a decentralized, trusted, and secure manner. We discuss stateof-the-art blockchain-based schemes, research projects, business initiatives, and case studies to highlight the practicability of blockchain in the oil and gas industry. We present the potential opportunities brought about by blockchain technology in various use cases and application scenarios. We introduce several systems that leverage blockchain-based smart contracts to automate the important services in terms of tracking and tracing of petroleum products, protection of international trade documents, and coordination of purchasing and bidding activities for granting oil exploration rights to petroleum exploration and development companies. Finally, we present open challenges acting as future research directions.
\end{abstract}

Index Terms-Blockchain; Oil and Gas; Traceability; IoT; Security

\section{INTRODUCTION}

Emerging technologies such as the Internet of Things (IoT), fog computing, cloud computing, and blockchain can play a vital role in boosting the operational efficiency of the oil and gas industry. Recently, a Russian oil company named Gazprom Neft has reported that digital technologies can increase the productivity of an oil and gas company by $10-15 \%$ [1]. The trading of oil and gas products such as gasoline and diesel is a highly standardized and quality-sensitive process that requires high security, privacy, and fast data processing. The majority of the systems developed to monitor and manage trade in the petroleum industry are centralized, unreliable, and highly nontransparent. Blockchain is a ledger of transactions that are secured using cryptographic hash algorithms and it makes the operations of the oil and gas users highly efficient, transparent, and trusted. A research study performed by Shell, BP, and Statoil has estimated that the adoptability of blockchain into

Raja Wasim Ahmad, Khaled Salah, and Ibrar Yaqoob are also with the Department of Electrical Engineering and Computer Science, Khalifa University of Science and Technology, Abu Dhabi 127788, UAE.

Raja Jayaraman and Mohammed Omar are also with the Department of Industrial \& Systems Engineering, Khalifa University of Science and Technology, Abu Dhabi 127788, UAE.

Corresponding Author: Ibrar Yaqoob (ibraryaqoob@ieee.org). the oil and gas industry can reduce the transaction execution time by $30 \%$ [2].

Digital technologies are aimed at transforming the oil and gas supply chain services, reshaping industry competition, ensuring the safety of workers involved in oil and gas wells development, enhancing the reservoir's resource usage rate and production potential, providing production forecasting across thousands of wells, boosting operational efficiencies and output of oil fields [3]. The IoT and machine learning can assist the oil and gas operators in providing a long-term forecast of the condition-based maintenance of oil and gasrelated equipment used in oil and gas exploration (e.g., oil wells drilling) to maximize its efficiency. Based on the type of the services, the oil and gas businesses are classified into upstream, downstream, and midstream sectors. The upstream service involves oil and gas exploration, extraction, or production of raw material such as crude oil from the wells. The midstream sector involves (a) transportation services for safely shipping the crude oil and natural gas using pipelines, tankers trucks, and heavy goods vehicles (HGV), (b) warehouse services for the temporary storage of crude oil at oil field terminals, and (c) refinement services to perform some initial processing of crude oil at the terminal. The downstream stage involves the refinement of the crude oil (at the oil refining facility) shipped from various oil production sites to make usable and marketable products such as gasoline, petrol, diesel, and Liquefied natural gas (LNG) [4], [5]. Many of the systems developed for automating the business processes in the upstream, midstream, and downstream sectors have followed centralized architectures to store and process oil and gasrelated data.

The centralized data storage and processing provide limited transparency to the data and operations of organizations and often results in data inconsistencies among the participating organizations of the oil and gas industry. As a result, centralized approaches offer limited collaboratiion opportunities to the stakeholders of the oil and gas industry. The systems that follow centralized data storage and processing are unreliable and less trustworthy as they are prone to data modifications and deletion by adversaries. Blockchain is an evolving decentralized technology that is highly capable of handling the trust, security, and cost-related issues in existing systems. Blockchain enables the existence of Bitcoin and many other cryptocurrencies. A cryptocurrency refers to a digital asset that works as a medium of exchange between various business organizations [6]. The digital assets are encrypted using state-of-the-art cryptographic hashing functions to adequately protect the tokens from external attacks, fully 
control digital token creation, and verify the transfer of digital tokens. Further, the employability of cryptographic hashing algorithms and digital signatures provide valuable help in avoiding double-spending of the same digital tokens [6], [7]. By design, blockchain follows a distributed Peer-to-Peer (P2P) architecture to store and process the data and transactions. The distributed storage and processing lead to a highly secure, reliable, and available system that satisfactorily safeguards the data from a single point of failure problem [8], [9]. Blockchain technology collects data and transactions in the form of blocks that are interlinked and assures data integrity.

A consensus protocol followed by the existing blockchain platforms assures that the most recently added block to the existing ledger is approved by the majority of verifier nodes within the blockchain network. The miner nodes within a blockchain network are responsible to validate the data and transactions and block creation [10], [11], [9]. Miners are rewarded with cryptocurrencies for their participation in the consensus process to verify and validate the transactions to create a block. Among many examples, Proof-of-Work (PoW) [12], Proof-of-Stake (PoS) [13], and Proof-of-Authority (PoA) [14] are three well-known consensus algorithms that are followed by numerous blockchain platforms [9]. PoW consensus algorithm requires the miners to satisfactorily solve a mathematical puzzle to form a block and claim the reward. The PoS selects a miner node to create a block based on several factors such as the total stake of the particular user or age. The PoA consensus algorithm counts the reputation score of the miners to select an appropriate node for mining a block. PoA is a highly fault-tolerant and computationally fast protocol [9]. Existing blockchain platforms are capable of providing better security, data immutability, improved accountability, and access control. Hence, such platforms can dramatically improve the services within the oil and gas industry to enrich the user experience [15]. Based on the architectural design, blockchain platforms are principally grouped into public, private, consortium, and hybrid blockchain platforms. A broader overview of the differentiating features of private and public blockchain platforms is presented in Table I.

The public blockchain platforms are open network of connected nodes that allow users to have full control on their transactions and data [16]. The users of the public blockchain platforms can access, view, update, and write data on the blockchain ledger without involving any third party. The private blockchain users follow an invitation-only model to access the platform. Private blockchain platforms are controlled and managed by a single entity. Permission is required from the designated entity by the users to join the network or access, view, and write data and transactions on the blockchain. In hybrid platforms, public platforms host private networks to control access to the blockchain platform. Through hybrid platforms, restricted access is granted to only a limited number of oil and gas industry users. The consortium or federated blockchain platforms involve several organizations and entities to control and govern the platform [17], [15], [9], [18]. The adaptability of a particular blockchain-based platform into the oil and gas industry is affected by the security and privacy requirements of participating organizations, blockchain de- ployment cost, platform scalability, and transaction execution throughput.

The challenges caused by outdated and non-transparent trading platforms being practiced by the oil and gas industry can be overcome by leveraging blockchain technology to improve supply chain operations such as planning, procurement, traceability, trading, marketing, and logistics services in the oil and gas industry [19], [20], [21]. More specifically, blockchain assists in securing and simplifying oil and gas trading, shipment tracking, inventory control, documentation, and billing and payments. It simplifies the unwieldy and complex oil and gas supply chain processes by introducing transparency to the involved business processes. To the best of our knowledge, only one survey study exists already that has discussed the applications and risks associated with blockchain adaptability in the oil and gas industry [22]. We conduct this study to investigate the role of blockchain in the oil and gas industry by exploring potential opportunities and applications, presenting several blockchain-based research projects and case studies, and discussing the challenges that need further research to improve the services of users in the oil and gas industry. The main contributions of this paper are as follows:

- We review and present insightful discussions on the existing blockchain-based solutions for the oil and gas industry. We report several blockchain-based research projects as case studies to demonstrate the practicality of blockchain technology in the oil and gas industry.

- We explore the key opportunities and applications of blockchain technology in the oil and gas industry to improve operational transparency, traceability, security, and accountability in supply chain operations.

- We discuss several blockchain-based systems for various oil and gas industry use cases to identify the system components, participants, role definition, and information flow among the users.

- We identify and discuss several open research challenges hindering the successful implementation of blockchain technology in the oil and gas industry.

The remainder of the paper is structured as follows. Section II reviews the state-of-the-art blockchain-based systems, business initiatives, and research projects focused on the oil and gas industry. Section III explores the opportunities and applications of blockchain in the oil and gas industry. Section IV identifies and discusses several open research challenges in the oil and gas field. Section V provides concluding remarks and future recommendations.

\section{A REVIEW OF BLOCKCHAIN-BASED SOLUTIONS FOR OIL AND GAS INDUSTRY}

In this section, we thoroughly review the existing blockchain-based solutions, case studies, business initiatives, and ongoing research projects put forwarded to assist the oil and gas field operators, owners, and regularities.

\section{A. Blockchain-based Solutions}

In [23], authors have considered a simple hashing algorithm; namely, SHA-1, for securing the transactions and monitoring 
TABLE I

COMPARISON OF PUBLIC AND PRIVATE BLOCKCHAIN PLATFORMS.

\begin{tabular}{lll}
\hline \hline Parameter & Private Platforms & Public Platforms \\
\hline Throughput & High & Low \\
Energy Consumption & Low & High \\
Level of Access & Single organization & Anyone \\
Scalability & High & Limited \\
Security & Limited & High \\
Ledger Type & Permissioned & Permissionless \\
Efficiency & High & Low \\
Consensus & Permissioned & Permissionless \\
Trust Among Nodes & Required & Not required \\
Transaction Execution Cost & Cheap & Costly \\
Identity & Identities are known & Anonymous \\
\hline
\end{tabular}

the petroleum products during their distribution. It securely records the data such as tanker operator identification (ID) code, identifiers of source and destination oil and gas servicing stations, liquid petroleum product quantity, and automobile location during the supply of petroleum-based products. The geolocation of oil tankers is estimated using a telematics technique that remotely monitors the movement of oil and gas products in real-time using a GPS-based module. The proposed system selects regulators, petroleum depot, filling station, and transporter nodes as the main system participants. It implements a consensus protocol that considers a transaction to be valid only if $75 \%$ of the network participants agreed to the current state of the blockchain. The proposed solution assists in protecting the transactions from external attacks. One of the limitations is that the geolocation data calculation and updating on the blockchain frequently can make the network slow by enlarging the ledger size.

The limited transparency of the operations performed by stakeholder organizations during the distribution of oil and gas-based products can affect the stakeholder's performance due to insignificant coordination and cooperation opportunities available to the involved stakeholders. In [24], the authors have proposed and evaluated a conceptual framework for the oil and gas managers and authorities who are involved in the decision-making process about oil and gas exploration and development. The proposed framework can assist the gas managers to investigate the legacy supply chain practices and suggest recommendations to integrate blockchain with the legacy systems to improve the operational efficiency of the existing industry. The proposed study has collected the data for analysis by following the judgmental sampling technique. The data has been collected from oil and gas managers from several refineries, oil and gas marketing companies, and oil and gas distributors. The collected data assists the organizations in investigating the effect of diverse factors on the operational performance of the legacy systems. Many factors such as the relationship with suppliers/customers, sub-contract management, just-in-time services delivery, and strategic planning, were considered to investigate the vital role of the aforementioned parameters in streamlining supply chain processes. The research concluded that blockchain has full potential to improve the operational performance of a company by integrating the existing systems.
An approach has been discussed in [25] that leverages blockchain technology to safely, timely, and reliably share data among the participating stakeholders in the oil and gas industry. The important data about the oil and gas resources, supply chain operations, and governance include registration of mineral rights, resource evaluation reports, exploration and development, production, situation analysis, and real-time monitoring of oil products shipment. A proxy re-encryption model has been employed by the proposed approach to further improve the data security and reliability features during business transactions execution among the stakeholders. The integrated proxy encryption technique can assist the stakeholders involved in data production to use the public key of the receiver to generate encrypted text. The receiver of the data can use its secret key to decrypt the encrypted message. Hence, such a method can enable the users in verifying that the original text is not altered by the network intruders. The proposed system has implemented a registration module for identity management, data manager to control data, and authority control smart contracts to define the rules related to user authentication, access control, and data sharing among different departments/organizations. Although the proxy reencryption technique protects the data sharing among the participants, it requires extra resources to process the data.

Based on the type of the operations such as drilling, exploration, transport, trading, and shipping, the oil and gas supply chain activities are mainly classified into three categories i.e. upstream, downstream, and midstream. Upstream involves exploration and production of oil and gas, however, midstream provides logistics, storage, and transportation services. On the other hand, the downstream services mainly deal with the storage and sales of oil and gas-based products such as petrol, diesel, and liquefied natural gas (LNG). In [26], a blockchainbased model has been proposed to automate the supply chain and logistics management operations in the Ecuadorian industry. It has used Hyperledger Fabric and Composer to develop the chaincodes for implementing supply chain management services of the oil and gas industry. The key stakeholders considered by the proposed model include gasoline supplier, manufacturer, distributor (tanker truck), retailer (also called gasoline station), and customer. The chaincodes have defined the rules that are followed during oil and gas logistics services to improve efficiency, productivity, and transparency. The 
TABLE II

AN OVERVIEW OF ROLE OF BLOCKCHAIN FEATURES IN OIL AND GAS INDUSTRY.

\begin{tabular}{|l|l|}
\hline Blockchain Feature/Property & Description of Role \\
\hline Transparency & $\begin{array}{l}\text { The transparency feature ensures that the oil and gas-related data and transactions stored on all network nodes } \\
\text { are consistent and can be viewed by all the authorized users. The transparency of operations in the oil and gas } \\
\text { industry increases the trust of the stakeholder organizations. }\end{array}$ \\
\hline Availability & $\begin{array}{l}\text { The decentralization and distributed storage characteristics of blockchain make it a highly capable technology } \\
\text { that ensures high availability of the data to the authorized users. The availability of data related to oil and gas } \\
\text { industry affairs can be helpful for the stakeholder organizations to make decisions efficiently and timely. }\end{array}$ \\
\hline Integrity & $\begin{array}{l}\text { The support of asymmetric key cryptography and timestamping to blocks assist the oil and gas industry stake- } \\
\text { holders to verify and protect the data and transactions. }\end{array}$ \\
\hline Data Provenance and Audit & $\begin{array}{l}\text { The consensus algorithms and hashing make blockchain an immutable technology. The unmodifiable data and } \\
\text { transactions is useful to analyze the performance of oil and gas industry users by the authorities. }\end{array}$ \\
\hline Authorization & $\begin{array}{l}\text { The digital certificates and identify management practices are helpful to assure authorization of oil and gas ind- } \\
\text { ustry stakeholders. }\end{array}$ \\
\hline Privacy & Data privacy is ensured by creating private channels among the participants in the Hyperledger Fabric platform. \\
\hline Pseudonymity & The identities of all the participating organizations in oil and gas industry are pseudonymous. \\
\hline Programmability & $\begin{array}{l}\text { All business affairs and terms between the participating stakeholder organizations can be automated through self } \\
\text { executing smart contracts. }\end{array}$ \\
\hline
\end{tabular}

proposed study has overlooked documentation management and its related frauds for cross-border oil and gas trading.

The existing systems leveraged to manage a network of oil and gas pipelines are facing several challenges related to automatic transaction execution, in-time warning issuing, and reliable data collection and processing. In [27], a system called Smart Micro-GaS has used many emerging technologies including blockchain and cloudlet (edge-based nodes) to effectively address the aforementioned challenges. The design and deployment model of Micro-GaS has focused on various industrial aspects such as multilevel, multiview, and multidimensional, to completely meet the requirements of the oil and gas industry operators. Blockchain technology can assist in securing the oil and gas-related data, and it autonomously triggers the notification for the authorities based on the analysis of collected data such as temperature and volume of gas in pipes. The smart contracts that are deployed by the Micro-GaS can enable the users to trade the oil and gas on the blockchain, and it assures that the available gas volume is immediately updated after the gas purchase transaction is marked successful.

\section{B. Research Projects and Case Studies}

A consortium of companies including the BP company, Mercuria energy group, and Shell created the Vakt platform to digitize numerous use case scenarios in the oil and gas industry. The minimum transaction settlement time, trusted auditability, fast reconciliation, and elimination of the manual paper-based system to record the trade-related transactions are few advantages of the Vakt platform. It assists the traders in handling post-trading processes which are often very slow, complicated, and unverifiable. It ensures that no default access is granted to the sensitive data, thus preserving the data privacy [28], [29]. Recently, IBM, Trafigura, and Natixis have developed a blockchain-based system for commodity trade finance for US crude oil transactions for enhancing data security and optimizing business efficiency [30]. The proposed system is built on Hyperledger Fabric and it has primarily focused on trade documents management, shipment tracking and state updating, and assurance of shipment delivery and payments. By using this system, shipping companies, pipeline operators, and inspectors, or warehouse operators can view and provide the updated status about the crude oil transactions in real-time. Thus, it results in minimum fraudulent transactions, and ultimately the degree of trust among users increases.

BHP Billiton aimed at employing blockchain technology for improving oil and gas supply chain operations, tracking and securing the data generated during product delivery, and creating and maintaining a chain of requests to connect the analysts, engineers, and laboratories. It also aims at developing a blockchain-centric platform for tracking the equipment or parts of the equipment used in the oil and gas industry and recording data about ownership transferring, maintenance history, and equipment reliability features throughout its lifetime. By analyzing such data the causes of equipment failure or performance degradation can be identified and fixed immediately. Recently, a prototype of a system has been developed by using the Rai Stones project to track oil wells bored samples recorded on the blockchain. Samples related to well boring are expensive and often lead to a heavy financial loss if not managed properly [31], [32]. Another business initiative called PermianChain Technologies is a blockchain-based startup that increases the efficiency of the activities involved in the supply chain of oil and gas-based products distribution. The platform is built on IBM's Hyperledger Fabric platform and it allows organizations to trade oil and gas products on the blockchain in a secure, private, and efficient way. Windermere capital is a Canada-based firm that has recently employed PermianChain technologies to tokenize their natural resources [33], [34].

ZiyenCoin is an SEC-compliant (Securities and exchange commission) token issued by the Ziyen energy company for trading several types of energy products in the oil and energy industry. The targeted system, called the Zayen platform, will support transferring of tokens between the stakeholders in the oil and energy industry and updating the owner of tokens. The potential users of the Zayen platform are the investors in energy-producing assets and operators who are responsible to develop renewable energy projects. Recently, the first version of the Zayen system was successfully deployed. The deployed platform has successfully recorded a transaction related to 


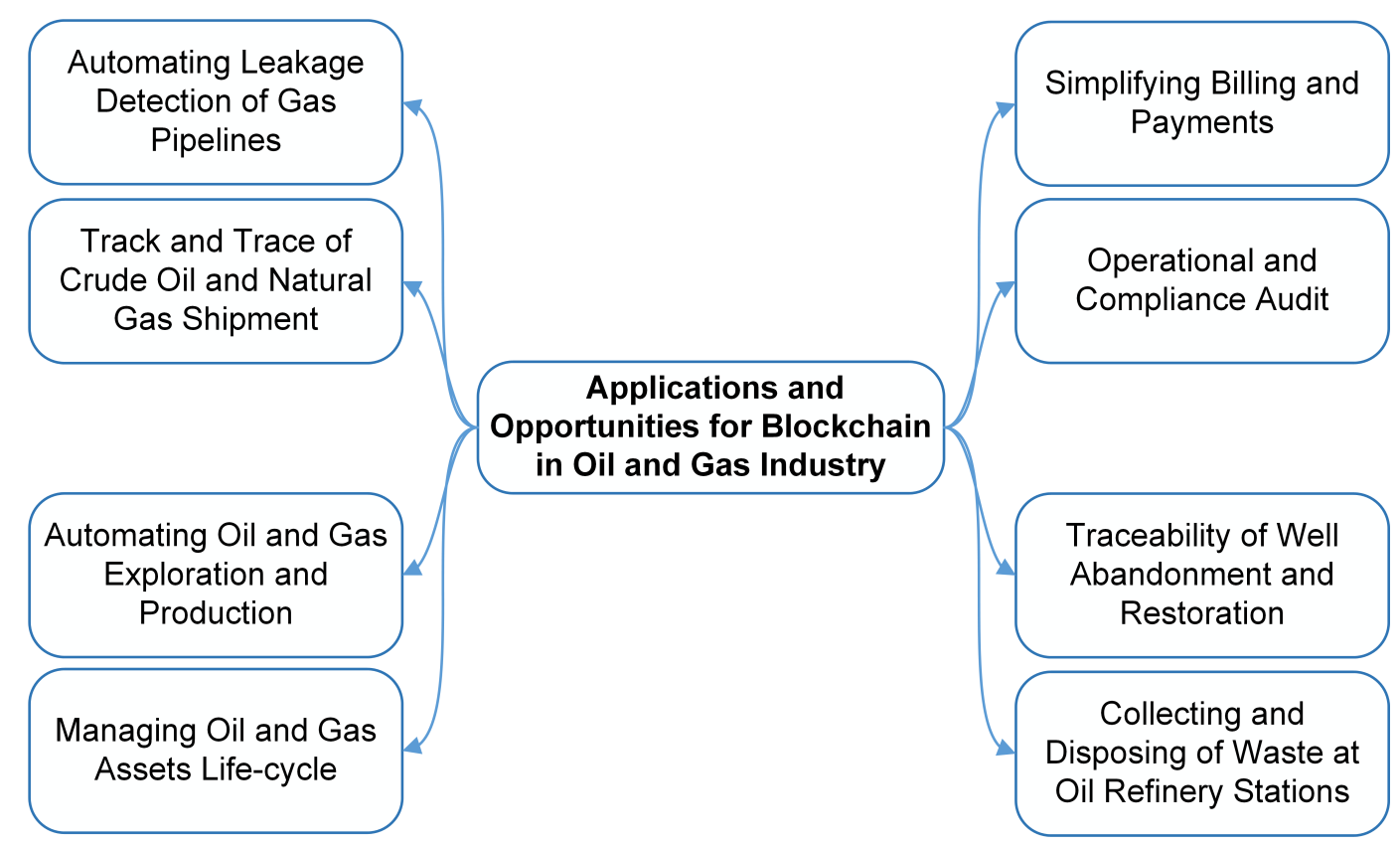

Fig. 1. An overview of the potential applications and opportunities for blockchain in the oil and gas industry.

the acquisition of 241 acres of oil and gas land on lease using 2300000 ZiyenCoins [35], [36]. Similarly, in late 2018, Venezuela's government has announced their oil and gasbacked cryptocurrency. The cryptocurrency was named Petro and it aims at assuring trade of the oil barrels on blockchain reliably, transparently, and securely. Also, PetroDollar follows US laws and represents a cryptocurrency that is backed by the oil and gas industry [37].

Bilur is created by a London-based company and it represents a digital currency that is backed by the units of stored energy. This digital currency system is enabled by a P2P network, distributed technology, PoS consensus, and publicprivate encryption mechanisms. The platform developed by Fintech allows users to trade energy commodities by selling and purchasing Bilur energy tokens in a decentralized fashion. In contrast to Petro cryptocurrency, Bilur is more transparent as its real value is visible to everyone and it is updated regularly. For instance, the current value of Bilur is equal to 6.481 oil barrels [37]. The UAE-based oil giant called Abu Dhabi National Oil Company (ADNOC) has collaborated with IBM to develop a Hyperledger Fabric-based system for automated accounting and fast transaction settlement during oil-based products sale. The ADNOC officials can use the pilot project to track the oil from the well to the customers to verify and validate the transactions. Such an analysis of transactions can assist in improving the efficiency of processes involved in the oil and gas supply chain [38], [39].

\section{OPPORTUNITIES AND APPLICATIONS OF BLOCKCHAIN TECHNOLOGY IN OIL AND GAS INDUSTRY}

Stakeholder organizations such as producer, shipper, supplier, and regulator actively involves in various stages of oil and gas trading from upstream to downstream. The issues of legacy systems such as lack of trust among participating organizations, low data visibility, and extended dispute resolution time can affect the performance of stakeholders of the oil and gas industry [40]. Table II provides an overview of the important blockchain features useful for improving the performance of stakeholders involved in supply chain and logistics management services of the oil and gas industry. Fig. 1 highlights the important opportunities and applications of blockchain in various sectors of the oil and gas industry. This section briefly discusses various use case scenarios, opportunities, and applications for blockchain technology to bring about trust, operational transparency, security, and faulttolerance to different sectors of the oil and gas industry.

\section{A. Pipeline Monitoring and Leak Detection}

The network of gas and oil pipelines enables the transportation of hydrocarbon fluids over large distances in the world. Pipelines are maintained safe as any pipeline surface leakage or damaging can lead to serious ecological disasters and human casualties [41]. The real-time monitoring of pipelines along with notification triggering about any theft, intervention, or leakage of the pipeline can assist to avoid such incidents. Such information can assist in timely detecting and locating the leakage of gas or even predicting the gas leakage based on the recorded data such as the current condition and total lifespan of the pipeline. The sensors are often attached to the gas pipelines to collect the pipeline's state data. The most practiced pipelines monitoring techniques and sensors aiming at pipelines leakage detection include pressure point analysis, infrared thermography, acoustic emission, ground penetration radar, and fiber optic sensor [41], [42], [43]. The existing pipeline monitoring and leak detection methods have followed centralized-based architectures for storing and processing on-field sensor data [41]. Thus, it can affect the reliability, availability, and security of the data that is used 


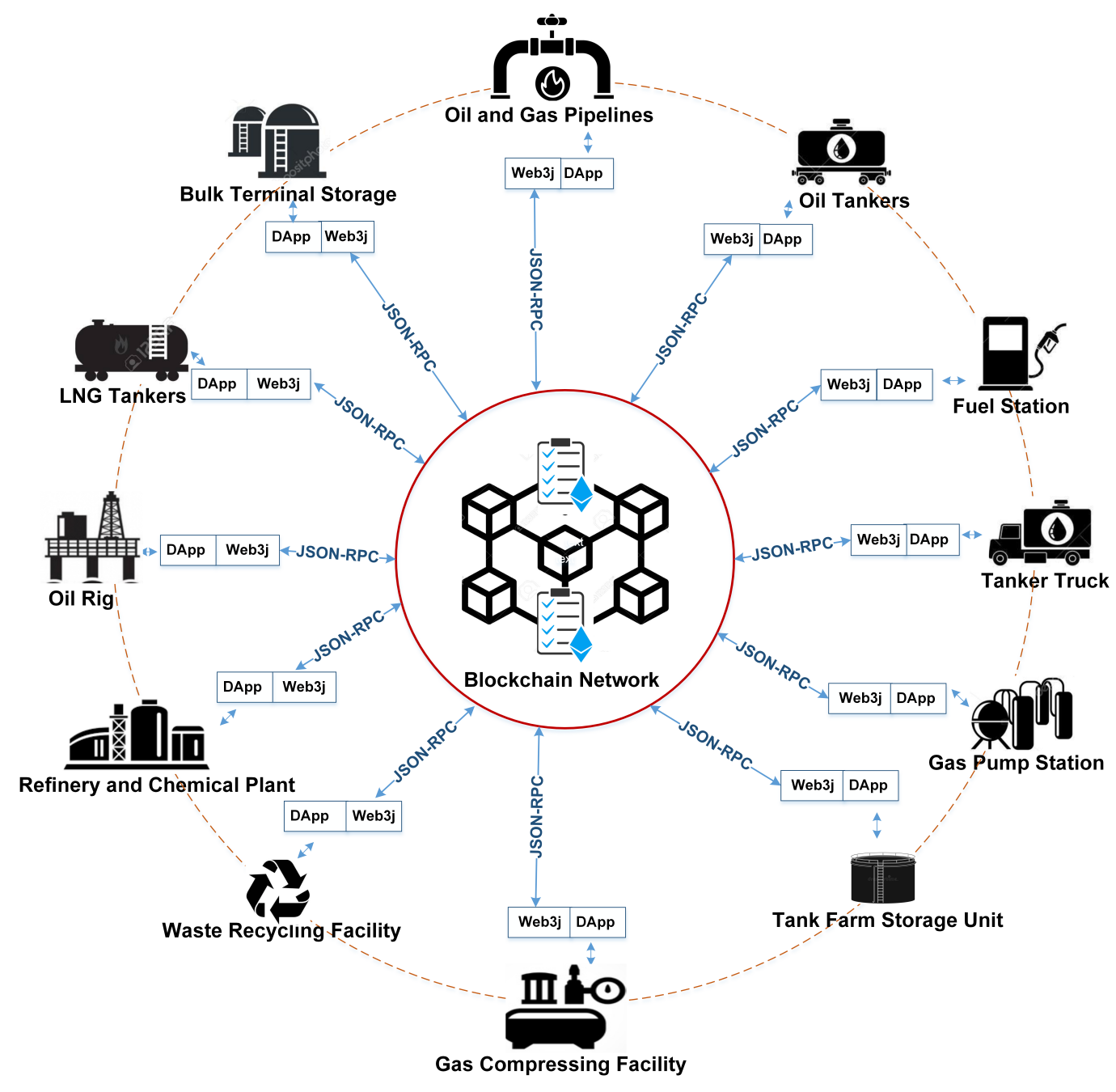

Fig. 2. An overview of oil and gas-related data storing and retrieving using the blockchain.

for pipeline leakage detection, prediction, management, and lifespan estimation.

Blockchain technology is capable of accurately detecting gas pipeline leakage and preserving the integrity of the pipelines. It can assist in verifying that the activities of the stakeholder organizations involved in the pipeline monitoring, leakage detection, and servicing are compliant with the human safety laws. It can assist in registering and verifying all onfield IoT-based sensing devices and aerial vehicles that are used to monitor the gas flow inside the pipeline to assure data reliability. As a result, the collected data by such sensors about the hydrocarbon fluids and pipelines will be highly trusted, verified, and reliable. Such data includes temperature, gas and oil pressure, and gas flow rate inside the oil and gas pipelines. Blockchain-based smart contracts can be developed to assist oil and gas companies in processing such data to precisely identify the gas leakage location in a pipeline [20], [44]. Through blockchain-based smart contracts, notifications can be immediately generated and sent to the pipeline operators on identifying any leakage issue along with sharing the location and identifier of the leaking pipeline for appropriate servicing. Also, the services provided by the pipeline operators to the leaking pipes along with the cause of pipeline leakage can be transparently recorded on the blockchain platform. Such data can be used by a performance smart contract to reward the off-shore service-providing organizations. For instance, the oil and gas companies can provide incentives to the workers based on the number of worked hours per day while servicing a leaking gas pipeline.

The stakeholders of the oil and gas industry which are involved in oil and gas pipeline management are supported by the blockchain to communicate in a timely, secure, and uninterrupted manner to increase oil and gas industry productivity. Fig. 2 provides an overview of a system that highlights system components, stakeholder organizations involved in oil and gas supply chain processes, on-field oil and gas-related entities and monitoring devices, and smart interfaces that enables the users to interact with each other through a trusted network. All these entities are restricted to communicate with the blockchain using JSON-RPC calls. Moreover, as can be seen in Fig. 2, smart contracts can be developed, deployed, and tested to efficiently automate the services within the various sectors of the oil and gas industry. 


\section{B. Tracing and Tracking of Oil and Gas Shipment}

Crude oil is moved from the wellhead to the refinery facility through pipelines, railroads, trucks, and sea routes. The petroleum products developed at refinery facilities are often shipped to industries or domestic users through tankers, railroad tank cars, trucks, or pipelines. On the other hand, because of high pressure and chances of explosion, tankers are not preferred to ship compressed natural gas. Instead, it is first converted into liquefied natural gas (LNG) and then it is shipped to the users using gas LNG tankers [45], [46]. Tracing and tracking services can assist the users in verifying the legitimacy of the data and assuring the compliance of actions with the rules published by the regularities for logistics and supply chain operations in the oil and gas sector [47], [9]. Also, through the tracking feature, the current location of oil and gas products, shipment state, and transportation route can be monitored, verified, and revised. The trace feature enables participants to identify, verify, monitor, record, and effectively manage the data about the users' operations and outcomes during oil and gas-based product shipment. The important data about oil and gas shipment processes include credentials about oil and gas shipment handlers, updates about shipment state and status, precise geo-location of tankers, estimation of the time for trucks arrival at the destination station, and congestion rate estimation on the routes.

The decentralization characteristic of blockchain technology makes it unmodifiable by external events. During the shipment of oil and gas-based products, all updates and changes in ownership are immediately recorded on the blockchain as these products cross the organization's border. Such records can be used to establish the data provenance of the oil and gas-based products to identify the frauds related to oil and gas products. Also, based on analysis of such data, smart contracts can be proposed for assisting the stakeholders to update the reputation score of the oil producers by analyzing the quality of supplied oil and gas-based products. Also, on experiencing the sub-standard oil and gas products, the blockchain can be consulted by the authorities (traceability) to identify the station where impurity was added to the oil and gas-based products and penalizing it accordingly. The traceability also assists in enforcing tariff and trading policies, preventing compliance violations, and identifying the root cause of delayed oil container shipment [9]. Tracking the location of the vessels that transport the giant oil tankers can assist port terminal authorities in preparing a resource-efficient stowage plan for the destination port terminal by assuring high productivity, performance, throughput, and risk mitigation [9]. The shipment tracking is performed by attaching the sensors to the trucks carrying the oil and gas-based products [48]. The sensors can also assist in verifying that any illegal attempt to open the oil and gas tankers can be immediately noticed. Any attempt of adding impurity to the shipping oil and gas tankers can be notified to the authorized organizations. Finally, the smart contracts [21] can assist the authorities in monitoring the state of the oil in tankers using various sensors that captures data related to temperature, volume, humidity, pressure, and container jerking.

\section{Automation of Oil and Gas Exploration and Production}

Oil and gas exploration and production (E\&P) is a costly phase as it involves oil and gas searching and exploration, drilling, and production from the drilled wells [10]. The exploration phase involves geologists who mostly follow seismology techniques to search the location of oil and gas reserves beneath the ground. On identifying viable oil and gas reserves, wells are drilled to test the quality and quantity of oil and gas reserves before starting the production process [25]. Engineers and geologists analyze the samples collected from the wells to estimate the quantity of oil and gas reserves. Engineers also estimate the number of wells to be drilled for oil extraction, and they decide drilling methods (horizontal or vertical) based on the analysis of the on-field collected data [49], [50], [51], [25]. The extraction process involves collecting the oil deposits from the well through the deployed infrastructure. The production process employs ICT-based solutions to extract the deposits of raw material from the earth. These deposits are processed to remove the impurities. Natural gas is often processed at an on-site location. However, oil is extracted at an on-site location and then it is temporarily stored and shipped to the refineries using pipelines [50], [52]. The legacy E\&P systems follow highly vulnerable centralized-based approaches to manage the data; but, any error or corruption to data may lead to a high exploration and analysis cost.

E\&P organizations often sign a contract with several external stakeholder organizations to search, drill, and produce oil and gas. Since blockchain offers a single, consistent, updated, and unified view of transactions and records, hence the involved organizations can share updated data in realtime about the processes, outputs, and potential challenges in the oil and gas E\&P phase. The modern oilfield uses several IoT-based sensors which collect data about the oil and gas production process such as fluid saturation, geological features, steam injection rate, temperature, drill rate, pressure, wellhead temperature, and resource usage rate, from the oil fields [53], [19]. In traditional systems, such data can be vulnerable to modification by competing organizations or hackers; but, blockchain ensures that data generated by sensors is immutable. Mostly, oil and gas E\&P organizations rent in the equipment from third-party organizations to drill oil and gas wells. Blockchain technology can enable organizations in automating the payments to the off-shore companies as a reward for their services in a fast, transparent, and trusted manner based on the equipment usage rate. Also, through private platforms, the trade secrets [54] of oil and gas organizations along with other sensitive data such as well logs can be protected. At the time of oil exploration, the generated data by the seismic surveys is very large; such data can be protected by generating and storing the hash of the data on the fault-resilient blockchain platform. Such data can be fed to AI-based algorithms to identify the data patterns. Also, drilling tools such as Logging while drilling (LWD) [55] collect and transmit the data to the surface for analysis. Smart contracts or chaincodes can be proposed for analyzing such data and identifying the abnormal behavior of data to highlight the root cause of the problem. 

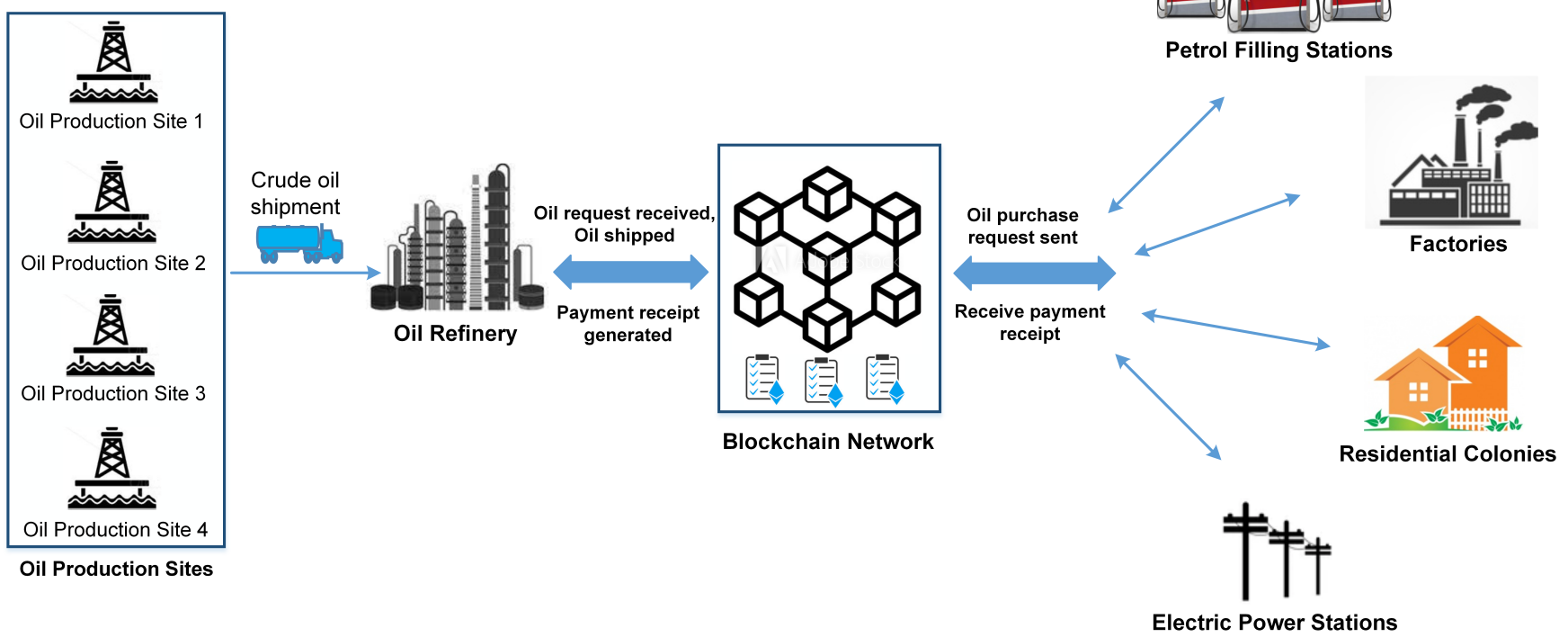

Fig. 3. Blockchain-based system for procurement and shipment of petroleum products.

\section{Management of Oil and Gas Assets Life-cycle}

The existing oil and gas companies own several types of assets which include machinery, equipment, and additional properties such as nitrogen production systems and oil treatment and refinement plants (OTP) [56]. Such assets are desired to be trusted and verified, thus they are required to be enlisted in the financial sheet of the oil and gas companies for assisting the authorities in assuring compliance with global or regional environment protection policies. Wellknown companies such as Petroleum (China), Technip FMC (UK), and Weatherford (Ireland) manufacture the equipment used in oil and gas exploration, production, and wells drilling. The primary equipment and machinery used in various sectors of oil and gas industry include tank vessels, heat exchangers, cranes and lifting machines, boilers, furnaces, compressors, oil and gas rotating equipment, and pressure vessels. The drilling of the oil and gas well involves up to 30 different service companies, thus requiring a common protocol that allows such companies to share real-time data in a secure and trusted way [56], [57], [58], [59]. E\&P companies procure equipment from reputed manufacturers when the demand for oil and gas equipment suddenly surges due to local inventory shortage. The oil and gas equipment's management-related activities such as global procurement, installation and infrastructure development, shipment through sea land, and repairment are very complex as they involve globally interconnected partners with different competing interests. The maintenance and repair services aim at restoring the reliability and safety of the oil and gas equipment. Existing systems used to manage oil and gas properties, machinery, and equipment are mostly web-based and centralized; hence stakeholder organizations have limited control over the supply chain processes.

Safety and conformity of equipment used in oil and gas exploration and well drilling can be increased using blockchain technology. The authorities can establish data provenance to ensure that oil and gas equipment used for E\&P operations comply with minimum safety standards. Through blockchainbased smart contracts, oil and gas companies can keep a track record of the available equipment and machines in the local inventory. Based on such data, the companies can proactively put a procurement request to the external organizations for equipment buy and sell processes after assessing the reputation score of the manufacturers or suppliers. Blockchain features greatly help the oil and gas equipment inventory manager to assure that the procured equipment are (a) shipped from a registered manufacturing company, (b) genuine, and (c) not fabricated beforehand [40], [9], [19]. The fabricated, uncertified, or substandard equipment/machines which are supplied through the gray or black market often require replacement or maintenance services, thereby increasing the equipment cost, workers safety issues, or unplanned downtime. The maintenance services are mainly classified into multiple categories such as predictive and condition-based maintenance services [60]. Blockchain can assist in locating the oil and gas equipment that requires Maintenance, Repair, and Operations (MRO) services based on their performance score. Blockchain can immutably record the maintenance history of the oil and gas equipment such as malfunction equipment ID, repairing station ID, repairing date, health score of malfunction equipment, and estimate of life competency of oil and gas equipment. The complete maintenance history of the equipment can be helpful to ensure compliance with safety rules during the wells' development, restoration, abandonment, and oil production operations. In addition to the maintenance history of the equipment, its operating history can be recorded securely on the blockchain [40]. 


\section{E. Simplifying Billing and Payments}

Recently, the trend of outsourcing independent contractors to drill wells in the oil and gas industry has been noticed as it decreases the operational and management cost [61]. For instance, an E\&P company can hire an oilfield equipment and services (OFS) contractor to rent in their equipment to drill an oil and gas well. Billing documents present the payment records made by an organization, and they are protected from modification as they serve as proof of payment during the dispute resolving between stakeholder organizations. A billing document essentially declares the amount payable to the service provider, payment due date, and records of account receivables. In supply chain management of the oil and gas operations, many types of billing documents are created and shared among the involved organizations [62], [63]. The oil and gas supply chain management processes such as procurement of crude oil by a company and refinement of crude oil to make products require creating, storing, updating, and managing multiple billing documents. It has been reported that the participating organizations in the oil and gas industry require up to 90 days to complete the payment process [62]. The main causes of such delays are dependency on a centralized system that is vulnerable to hacking, human involvement requirement for business settlement, and nontransparent systems. Also, the existing centralized billing processing systems are not capable enough to handle micropayments efficiently.

The traditional oil and gas management systems assure the making of payment by the oil companies to the corresponding parties through close monitoring of an agreed-upon external event to calculate the payments due date. In many cases, the oil and gas companies follow a specific event to make the payment within 30 days of the receiving of the bill of lading (BOL) document [62], [19]. However, in a few other cases, the invoice payment due date is calculated using business or calendar days. The decentralized and immutable blockchain technology is capable of solving some of the issues of legacy centralized solutions regarding payment management. The traceability and transparency features of the blockchain technology can assist the oil and gas companies in verifying that the payment of a company was sent, received, and updated correctly by the corresponding organizations. It assists the stakeholders in assuring that the payment-related operations of the organizations are fully compliant with rules which are declared in the contract. For instance, oil and gas organizations involved in the oil refinery and shipment services can issue the payments upon receiving the commodity from the providers. Blockchain-based smart contracts can be developed and deployed to invite the participating organizations to deposit an escrow amount (twice of the commodity cost) in the smart contract wallet [64], [65]. This escrow value forces the participating organizations to behave honestly. The price of the commodity is immediately transferred to the wallet of the service provider (i.e., Petrol selling company) as soon as the commodity is received by the customer/dealer. The smart contract transfers the remaining cryptocurrency tokens to the wallet of the customer/dealer.

Blockchain support of cryptocurrency empowers an oil and gas organization to make micropayments directly to the account of other stakeholder organizations; hence, it offers a secure and transparent system that does not involve mediators to settle payment-related disputes. Also, in various jointventures of oil and gas companies, the trusted blockchain can be used to verify, calculate, and pay the joint interest billings [40], [19]. Fig. 3 presents a blockchain-based system that enables oil refinery facilities to sell and ship oilbased products to customers such as petrol filling stations, factories, residential colonies, and electric power stations. In the presented system, the deployed smart contracts can enable the users to request, verify, and ship oil-based products such as petrol, and requiring the customers to make payment on receiving the shipment.

\section{F. Regulation Compliance and Accountability}

Regulation compliance affirms that the operations of stakeholder organizations in the oil and gas industry should comply with state, regional, and global laws. The authorities are required to propose appropriate standards, monitor the organization's actions for assurance of compliance with set standards, and issue penalties or incentives to ensure enforcement. The penalty issued could be anything related to a financial loss or working license cancellation and it depends on the severity of non-compliance operation. The regulatory authorities such as the Bureau of Land Management (BLM) and the Environmental Protection Agency (EPA) can perform an on-site visit to an oil development plant for compliance monitoring and issuing penalties on non-compliance with standard operating procedures during operations. In many states, the oil and gas companies are obliged to secure a bond that is made payable to the government to start the oil exploration [66], [67], [68]. Also, it is required that the hydraulic fracturing chemicals used to crack the oil reserves should be safe for human health. It is also desired that the oil and gas companies should keep a detailed record of the petroleum payments made to the government or other entities for auditability purposes by the third parties. The existing systems employed to manage the data necessary for compliance monitoring lacks in providing data transparency, visibility, security, privacy, and fault tolerance.

Blockchain technology can assist the state agencies and authorities in compliance monitoring to the published standards to safeguard the humans and environment. The security, supply chain traceability, and data immutability features of blockchain can bassist the authorities to identify the type of chemicals that are used in the hydraulic fracturing process by the E\&P companies. There are many types of chemicals available to perform hydraulic fracturing, but few of them are dangerous to human health and their usage is banned globally. Using transparency to the blockchain records, the authorities can access the data and transactions to verify that banned chemicals are not used by the operating oil and gas company. It issues penalties to the oil and gas operating companies on identifying the usage of hazardous and substandard chemicals during the hydraulic fracturing process. Smart contracts can enforce the rules about bond securing by the E\&P companies from the government to drill an oil and gas well. The noncompliance with such rules is considered a violation that will 


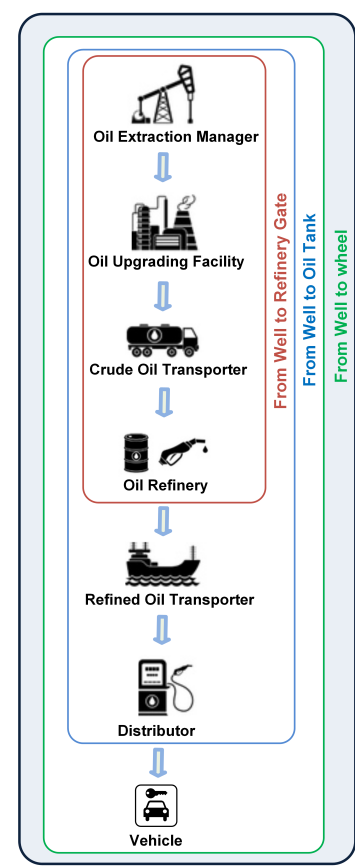

Stakeholders

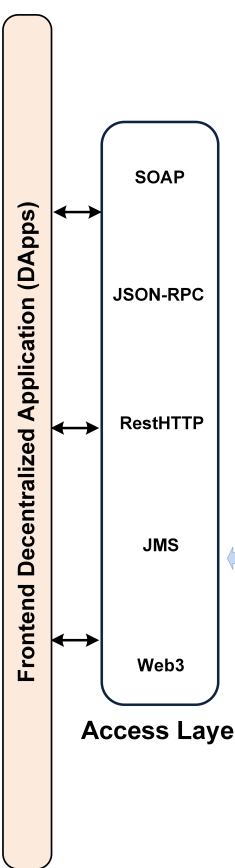$$
\int
$$

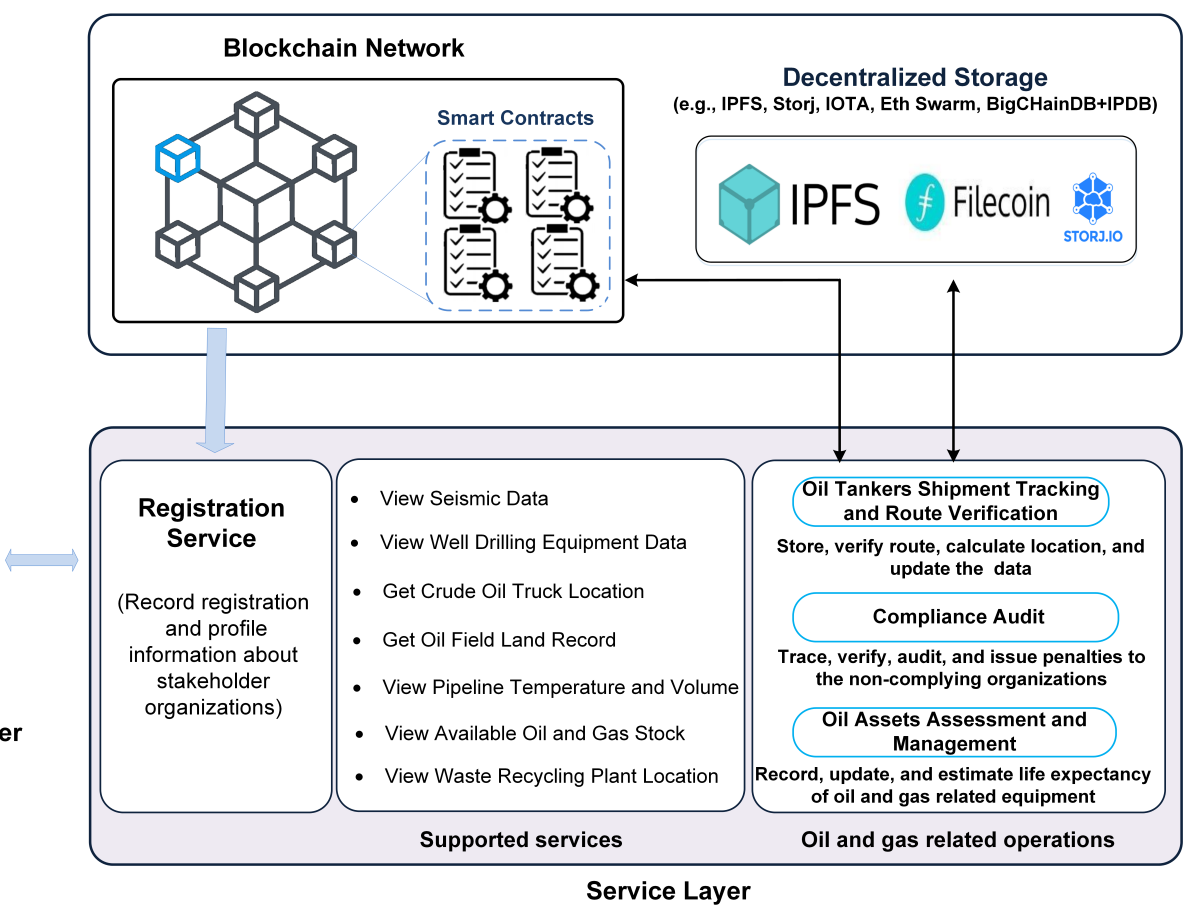

Fig. 4. Blockchain-based system for oil and gas supply chain operations.

result in a huge financial loss or license canceling. Also, the performance-monitoring smart contracts can be deployed on blockchain to verify that the operations of the users involved in the drilling of well, crude oil production and shipment, and oil and gas-based products distribution meet the desired criteria. The crude oil is often shipped in large-sized containers that are transported through sea mode. The international trade laws require the trading oil and gas companies to sign the paperbased trade documents before leaving or receiving the crude oil containers.

Fig. 4 presents a high-level design of a blockchain-based system that can be used by the oil and gas authorities or citizens to trace the crude oil from the well to the wheel of a vehicle. Blockchain technology increases the trust of the oil and gas traders by protecting the trade documents. For instance, a Bill of lading represents an acknowledgment receipt that is often provided by the shipment carrier to the exporter and it serves as proof of the crude oil shipment. Unlike the traditional systems which are extremely slow in processing trade documents, blockchain assure that trade documents reach the destination before the reaching of shipment. Using blockchain, authorities can trace, verify, and establish the origin of oil cargo. Such data will be helpful in the identification and prevention of smuggled oil cargoes. A blockchain-based system, as presented in Fig. 5, can enable the authorized users to create, sign, share, and verify the bill of lading document to speed up the crude oil delivery process. In the presented system, the oil carrier creates the bill of lading on the blockchain by storing the data such as owner and receiver of the shipment, details about the carriers, payments, and shipping items. After its creation, it is shared with the shipper (Refinery facility) to verify and sign it before giving it to the consignee. At the destination side, as can be seen in Fig. 5 (step 8), the document is checked and verified by the carrier before providing the shipment to the consignee.

\section{G. Traceability of Wells Abandonment and Restoration}

The oil and gas wells' abandonment and restoration is the final stage of the oil and gas development process. The temporary wells-abandonment type involves the removal of well control equipment from the oil field and assuring that the oil and gas extraction process will be resumed in the future [69]. The permanently abandoned oil and gas wells are never being re-used or re-entered; hence, they require urgent restoration. At the time of oil and gas wells abandonment, it is assured that the reservoirs are properly isolated to stop the flow into other reservoirs. After the abandonment of the oil and gas wells, it is restored to the pre-development state to minimize the environmental effects of the oil development process. The main processes involved in oil and gas wells restoration operations include soil and water sample collection, disposal of concrete, well pads and trash, site leveling, and removal of radioactive materials [69], [70]. The data relating to oil and gas wells' abandonment and restoration is often stored, processed, and managed by untrusted and costly systems.

During the oil and gas wells abandonment and restoration phase, the blockchain-based alert system with the support of predictive AI analytics can notify the workers about the disaster or a certain event that requires an immediate response. Blockchain can also assist to facilitate the clean-up of the oilpolluted land by monitoring the performance of the community members and issuing incentives as a reward to their services in a trusted and reliable way. After the land restoration phase, the new owner of the titled land can be updated and notified 


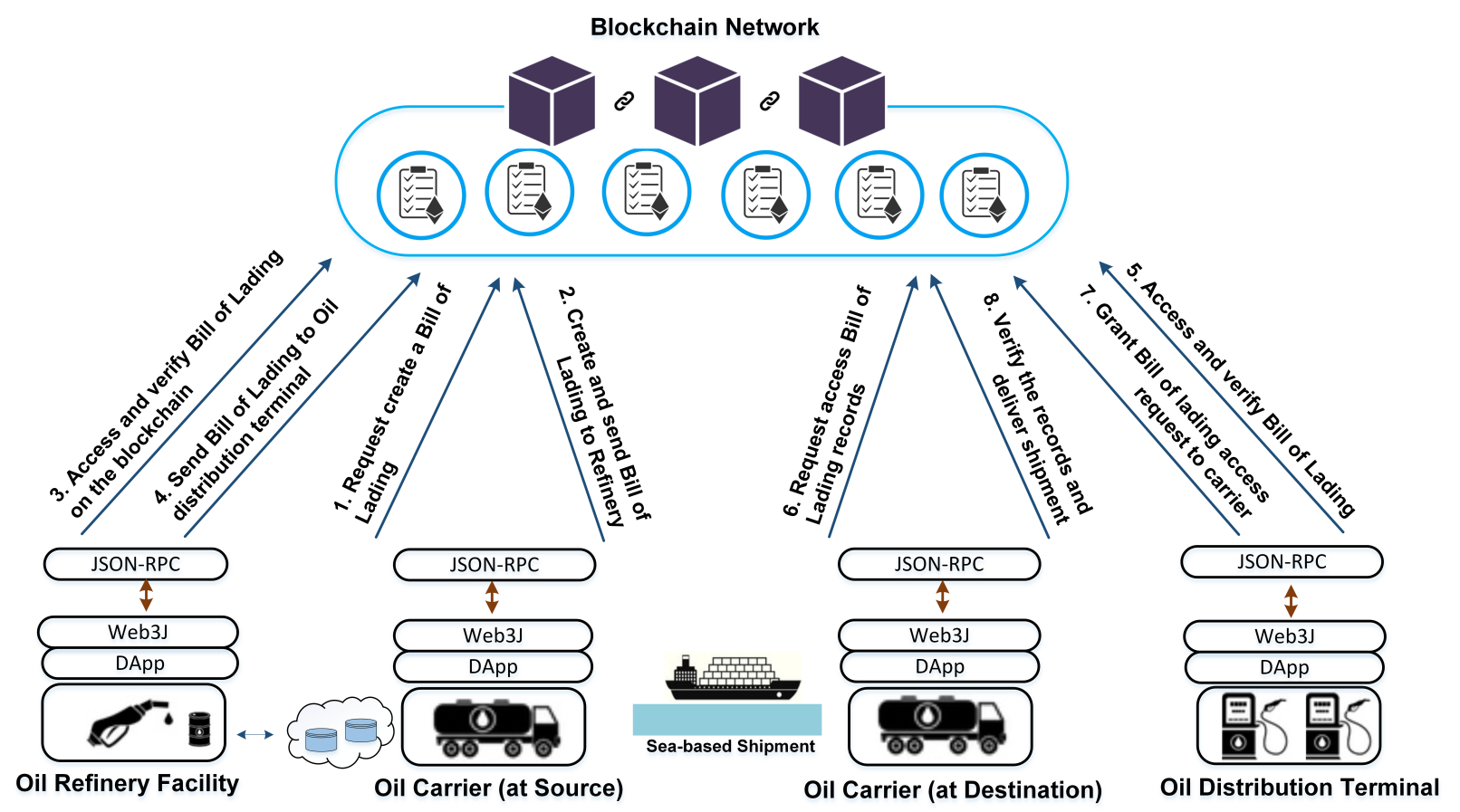

Fig. 5. Automating Bill of lading trade document using blockchain-based smart contracts.

to the concerned authorities. Blockchain-based data such as the owner of the wells, cause of abandoned oil well, type of abandonment, total explored or available oil reserves in the well, and safety measures that are taken into consideration during the oil and gas well's restoration are useful for the authorities to verify the records and operations. Such data can be used in making smart decisions about reusing such land in the future for other purposes such as crop cultivation. Fig. 6 presents a system that enables government agencies to successfully execute a bidding process to issue oil and gas wells drilling rights about the restored oil well to the winning companies. The auction contract is responsible to collect the bid amount of several oil companies, verify the deadline, and use the collected data (after deadline expiry) to declare the winner.

\section{H. Disposal and Recycling of Oil and Gas industry Waste}

The oil and gas sector generates various types of waste including wastewater, drilling fluid chemicals, drilling muds (sludge) and the toxic gas, and smoke. Some of these waste materials are recycled at a recycling plant, whereas the remaining waste is landfilled to protect the environment from harmful gases. According to environmental protection laws, the waste produced by the oil and gas industries should be properly treated before disposing of it. Tracing and tracking the waste in the oil and gas industry assist authorities to assure that ethical practices are followed by the waste handlers and processors during collection, segregation, shipment, treatment, recycling, and landfilling processes [71], [72], [48]. For instance, through IoT-based location service, the real-time location of the drilling fluid chemicals-related waste can be tracked, and further, it can be assured that waste was shipped to a designated waste recycling center [72]. The traceability feature can enable the authorities to verify that the generated waste at oil and gas processing stations is handled in compliance with the guidelines issued by the governments. The guidelines about waste handling mechanisms are given to the industry through certificates and documents that state the rules and methods to be followed during the waste handling, shipment, treatment, and disposal. Today's systems leveraged for waste management in the oil and gas industry offers limited trust, security, fault-tolerance, and dependability.

Blockchain technology assists users in minimizing wasterelated fraud (e.g., illegal dumping and shipment) by immutably storing the transactions and data on the distributed ledger. Smart contracts can assist in significantly minimizing waste-related delays, protect the documents from forging, and assure high data consistency. The tracking and tracing features can assist the organizations to verify the current location and route of the trucks shipping the industrial waste [73], [8]. The immutable transactions, data provenance, and smart consensus algorithms improve the trust between the stakeholders involved in waste management of the oil and gas industry. Blockchain also assists in timely, secure, and eventbased transferring of incentives to the wallets of the waste collectors, segregator, or shippers. The auditability feature, immutability, and provenance of data can assist the authorities and government agencies to assure that the rules, standards, and safety methods have been followed during waste handling practices. Also, blockchain can be leveraged to assure that all generated waste at crude oil refinery stations has been collected, processed, and landfilled properly. The records of the transactions can be used to verify the amount of waste lost during waste shipment, treatment, landfilling, or segregation. 


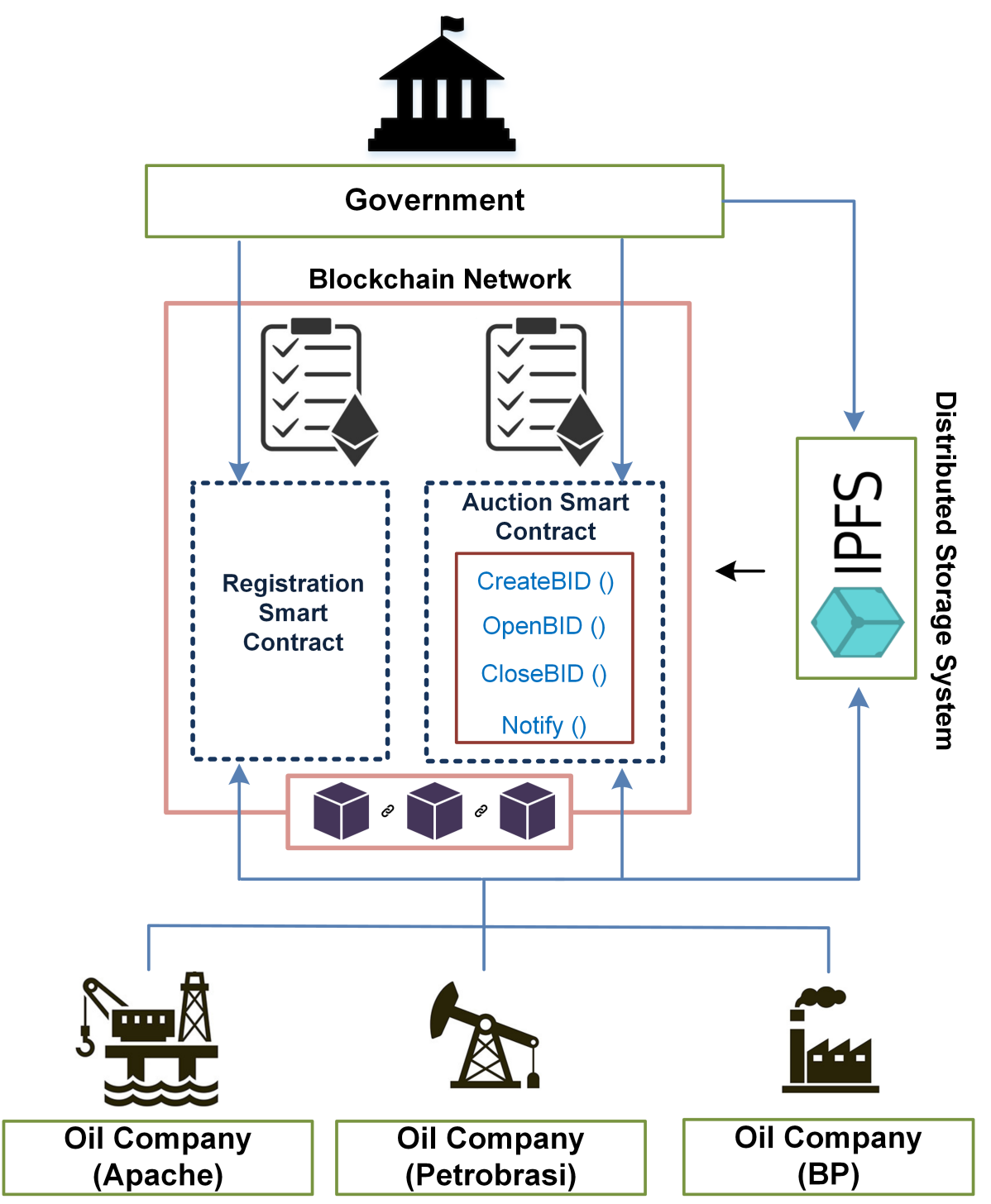

Fig. 6. Ethereum-based system for issuing oil and gas exploration rights to the oil and gas companies.

The responsible organizations can be identified and punished by issuing financial penalties to them. The authorities can also verify that the wastewater of the oil and gas industry is properly treated before it is reused for agricultural purposes.

\section{OpEn RESEARCH CHALlenges}

This section presents the open research challenges that hinder the implementation of blockchain in the oil and gas industry.

\section{A. Data Security and Privacy}

Blockchain technology employs resource-efficient consensus algorithms and irreversible hashing-based data encryption methods to secure the data and transactions related to oil and gas exploration, development, shipment, and other business operations in the oil and gas industry. The existing security policies and tools followed by the majority of blockchain platforms protect the user's data against malevolent and unintentional threats by detecting, preventing, and generating a strong response to such threats [74], [65]. For instance, by following the policy to choose the longest chain as a final and authentic chain, $51 \%$ majority attack and forking problems of blockchain technology can be successfully solved [75]. Data privacy enables organizations to transact on the blockchain without exposing their true identities. The existing blockchain platforms allow pseudonymity which refers to a state of disguised identity. But, there could be many application scenarios in the oil and gas sector that require the true identity exposing of the participating organizations [76]. As a result, the privacy of users' data will be affected significantly, and it can lead to regulatory and legal problems.

Generally, data integrity, availability, and confidentiality guaranteed by the blockchain platforms can assist in preserving the privacy and security of the data related to the upstream, downstream, and midstream oil and gas industry users. The data stored on the public blockchain platforms, such as Bitcoin, are more susceptible and sensitive to users' data privacy leakage problems. Private and consortia blockchain 
platforms (e.g., Hperledger Fabric [77], Hyperledger Besu [78], and Quoroum [79]) are suitable for many applications of the oil and gas industry as they preserve data privacy and assure fast transaction execution speed. The self-sovereign identity management of blockchain technology assists the users to preserve the privacy and security of data that is stored during the business processes execution among various users. The recently developed ZK-SNARKs protocol (ZeroKnowledge Succinct Non-Interactive Argument of Knowledge [80]) is a data privacy protection solution that is developed by the zCash and Ethereum jointly. Such a solution can be helpful for oil and gas organizations to meet the privacy requirements of oil and gas industry users. Further research in this domain should focus on proposing resource-friendly, reliable, trusted, and fast-converging time-based privacy-preserving solutions.

\section{B. Scalability}

The IoT-based sensors deployed in an oil field enables the oil and gas industry operators to closely monitor (a) the performance of equipment used for oil and gas wells drilling, (b) quality of materials used for oil extraction, and (c) efficiency of the system used to filter the extracted oil. The sensing devices used for monitoring such equipment and materials can generate a large amount of data as these sensing devices continuously sense, collect, and transfer the data to the blockchain. Usually, the stakeholder organizations involved in the oil and gas business operations require high quality of services (QoS) to meet their business goals [9]. The QoS offered by the existing blockchain platforms can be characterized in terms of transaction execution speed, throughput, latency, jitters delay, and transaction execution cost. The technical scalability of a blockchain platform is one of the major issues that significantly affect its adaptability to the users of oil and gas industry. The legacy unreliable systems such as Paypal and Visa are very fast. Visa is capable of handling more than 2000 transactions per second [76], [65], [9]. Blockchain-based systems are far behind such systems in terms of transaction execution speed.

The classical Bitcoin blockchain network can execute three to seven transactions per second, and it needs ten minutes on average to complete and publish a block. Employing Litecoin and Bitcoin cash can assist in effectively handle the Bitcoin scalability issue [81]. Also, through off-chain transaction execution in Bitcoin, the throughput of the system can be significantly improved. Similarly, the Ethereum blockchain platform (e.g., Ethereum 1.0) can execute up to twenty transactions per second. The Ethereum 2.0 promises to support more than 100,000 transactions per second to meet the requirements of oil and gas industry partners [9]. The private blockchain platforms do not suffer from scalability issues as they are purposely designed to allow a limited and trusted group of users to communicate effectively; but, with more centralized control. Sharding [82] is one of the solutions that clusters the transactions based on the computational requirements into shards by giving the freedom to nodes to not process every transaction in the history of blockchain. Thus, it minimizes the transaction execution time to successfully meet the QoS requirement of oil and gas industry users.

\section{Interoperability}

Many blockchain networks are not inherently open and do not support cross-platform communication between organizations. Hence, such blockchain platforms are disconnected and often presents silos of information; thus preventing oil and gas organizations from reaching their full potential. The cause of disconnected blockchain networks includes the difference in (a) hashing algorithms, (b) security protocols, (c) transaction type and format, and (d) consensus protocols. Blockchain interoperability support enables oil and gas-based organizations to see, share, and access data consistently across various blockchain-based platforms. It is beheld that organizations involved in oil and gas exploration and development (using heterogeneous platforms) often share cryptocurrency tokens. Several approaches are developed for blockchain interoperability problems to assure cross-platform communication among stakeholders to increase system throughput and minimize transaction processing time [83], [84].

Zendoo is a protocol that promotes interoperability support among blockchain-based platforms to empower oil and gas companies to send and receive cryptocurrency tokens on separate platforms. Mashup application is another solution for the interoperability problem of blockchain that provides a set of APIs to allow users of two heterogeneous platforms to communicate with each other smoothly. The majority of existing blockchain interoperability techniques rely on sidechains, notary schemes, and timed hash-locks for cross-chain communication between public platforms. Also, in a few cases, a completely separate blockchain or non-blockchain platform is used to allow the organizations to communicate with each other. Based on such techniques, interoperability projects such as Chainlink, Cosmos, Hybrix, Polkadot, and Wanchain are in the development stage to allow cross-chain communication among users without any intermediary third party [84], [65], [9]. Considering the requirements of the oil and gas industry stakeholders, the interoperability solutions should be secure, fast, fault-tolerant, and privacy-preserving.

\section{Smart Contracts Security}

The blockchain-based systems leveraged for oil and gas exploration, well development, and crude oil shipment rely on self-executing smart contracts. The real-time monitoring of crude oil shipment, well drilling equipment performance monitoring, equipment life competency management along with MRO history management, refinery facility service audit, and protection of trade documents are a few examples of smart contracts used to digitize and automate the oil and gas industry services. The code of a smart contract automatically executes when a predetermined criterion agreed by the participating organizations is met. Smart contracts implement rules which are implemented in an appropriate programming language. Such contracts can be executed on the underlying blockchain platform as they have support for them. For instance, the Ethereum blockchain platform supports smart contracts written in Solidity language. By nature, smart contracts are deterministic and become immutable when they are deployed on the blockchain by the users. The performance and trustworthiness 
of smart contracts highly depend on their defense system against external and internal attacks. Any bug or error in a smart contract can cause considerable losses to the accounts of stakeholders. For instance, in 2016, an Ethereum smart contract hacked by the hackers resulted in 3.6 Ethers loss [85], [86], [87], [65], [9].

Therefore, smart contracts are required to be carefully written, tested, and verified before their deployment on the blockchain to avoid their exploitation by hackers for malicious activities. Smart contracts testing tools such as SmartCheck, VeriSolid, Gasper, Oyente, and Securify statically analyze the code, and they are capable enough to provide guidelines for performance, cost, and security enhancement of smart contracts. Similarly, the smart contracts security analysis tools including Vultron, Regaurd, EasyFlow, and ContractFuzzer have followed the dynamic analysis approach. Such tools can greatly assist the developers in identifying the bugs of various types including integer overflow and underflow, non-validated arguments, default visibilities, prodigal contracts, and block gas limit in the smart contract code [88], [87], [65]. The badly written and tested programs are not trustworthy and they can affect the adoption of blockchain technology by the stakeholder organizations of the oil and gas industry.

\section{E. Blockchain Deployment Cost and Slow Adoption}

Blockchain has shown the huge potential in bringing trust, transparency, security, and audit services to the oil and gas operations for automating the exploration, development, production, refinement, auctioning, and shipment of oil and gas. The novel characteristics of blockchain technology and its successful integration with existing systems such as distributed file storage systems can create new opportunities for the competing oil and gas companies; thus, it further increases the trust among the participating organizations. It also assists the oil and gas companies in improving the business services about automating rig plant, LNG tankers truck, bulk terminal storage facilities, fuel filling stations, and waste management facilities. The energy consumption due to computationally expensive mining processes and gas consumption rate of the smart contracts are the main drivers of the total deployment cost of the blockchain solutions [89], [90], [65], [9]. In a blockchain-based solution, gas cost refers to the amount of fee that is paid by the users to the block creator as a reward for mining service. However, since private blockchain platforms are owned by a single organization, hence they do not involve gas consumption during smart contract execution.

In Ethereum-based blockchain pltaform, the gas consumption of a particular function in a smart contract is estimated based on consumed gas units during function execution and total gas price. The gas consumption of a smart contract depends on the code size, type of operations within the functions, the complexity of operations, and arrangements of functions and data. The gas price is not fixed and it is dynamically calculated by estimating the resources consumed by the smart contract functions when they are called [90], [65], [9]. Despite bringing many advantages to the oil and gas industry, the adaptability of blockchain is still low because of many industrial factors and the incompetencies of blockchain developers. For instance, the involved stakeholder organizations may have shown limited interest in shifting their business processes to the blockchain. Moreover, as blockchain is still in its fantasy stage, hence blockchain standards and governance rules are not mature enough. The unavailability of blockchain developers and regulations is another reason for the slow and limited adoption of this technology by the oil and gas industry users [90], [91], [9].

\section{Concluding Remarks and Future RECOMMENDATIONS}

In this paper, we have discussed how blockchain technology can play a vital role to promote oil and gas exploration, production, refinery, and logistics services in a transparent, traceable, trusted, accountable, and secure manner. We reviewed and discussed the recent blockchain-based systems, industrial case studies, business initiatives, and ongoing research projects to highlight the practicability of blockchain in the oil and gas industry. We explored the potential opportunities and applications of blockchain technology in various sectors of the oil and gas industry such as real-time leakage detection of gas pipelines, tracing and tracking of crude oil and natural gas shipment, management of oil and gas assets life-cycle, traceability of well abandonment and restoration, and automation of billing and payments. We presented the high-level design of several blockchain-based systems to automate the business operations involved in tracking and tracing petroleum products, protection of international trade documents, and coordination of purchasing and bidding activities for granting $\mathrm{E} \& \mathrm{P}$ rights to petroleum exploration and development companies. We identified and discussed several challenges that require further research to unlock the full potential of blockchain in upstream, midstream, and downstream sectors of the oil and gas industry. Our concluding remarks along with some key future recommendations are as follows:

- The successful adoption of blockchain technology into the oil and gas industry is affected by many factors such as immature and globally unaccepted blockchain standards, legal and regulatory frameworks for blockchain, and its high implementation cost.

- The mining process of the PoW consensus algorithm needs a large amount of energy and computing resources in unlocking the mathematical challenges to mine a block. As a result, it leads to increased carbon dioxide emissions in the atmosphere. The mining-related energy consumption of the POW can be reduced by lightening the blockchain network.

- Maintaining the data related to oil and gas products trading using public blockchain platforms such as Ethereum enhances the security, reliability, and integrity of data. However, fulfilling the fast data storage and processing requirements of stakeholder organizations involved in the oil and gas E\&P process using public blockchain platforms is challenging.

- Leveraging the public blockchain platforms by the oil and gas organizations to automate the supply chain operations 
can affect the privacy of the participating organizations. The GDRP-compliant use cases and applications in the oil and gas industry can be proposed to preserve the user's data privacy using blockchain technology.

- Oil and gas is a regulated industry that operates under the strict rules and guidelines issued by the state and government agencies. Through blockchain, regulators can monitor the oil and gas exploration and production data in real-time, and they can issue heavy fines for noncompliance with worker's safety, land access, wastewater processing, and environmental protection laws.

\section{ACKNOWLEDGMENT}

This publication is based upon work supported by the Khalifa University of Science and Technology under Awards No. RCII-2019-002, Center for Digital Supply Chain and Operations Management.

\section{REFERENCES}

[1] T. Antipova, Integrated Science in Digital Age 2020. Springer, 2020.

[2] Yuri Musienko, "How to use blockchain in oil and gas industry," [Online]. Accessed on 22/04/2021, May 2019, https://merehead.com/blog/use-blockchain-oil-gas-industry/.

[3] Dam Ogji, "Digital transformation: Powering the oil and gas industry," [Online]. Accessed on 25/04/2021, August 2018, https://www.ogj.com/home/article/17297879/digital-transformationpowering-the-oil-gas-industry.

[4] TriStone Holdings, "What Are The Differences Between Upstream, Midstream And Downstream Oil Activities?" [Online]. Accessed on 25/04/2021, July 2020, https://tristoneholdings.com/oil-activities/whatare-the-differences-between-upstream-midstream-and-downstream-oilactivities/.

[5] A. Gezdur and J. Bhattacharjya, "Digitization in the oil and gas industry: Challenges and opportunities for supply chain partners," in Working Conference on Virtual Enterprises. Springer, 2017, pp. 97-103.

[6] M. B. Hoy, "An introduction to the blockchain and its implications for libraries and medicine," Medical reference services quarterly, vol. 36, no. 3, pp. 273-279, 2017.

[7] K. Yeow, A. Gani, R. W. Ahmad, J. J. Rodrigues, and K. Ko, "Decentralized consensus for Edge-centric Internet of Things: A review, taxonomy, and research issues," IEEE Access, vol. 6, pp. 1513-1524, 2017.

[8] R. W. Ahmad, K. Salah, R. Jayaraman, I. Yaqoob, M. Omar, and S. Ellahham, "Blockchain-based forward supply chain and waste management for COVID-19 medical equipment and supplies," IEEE Access, vol. 9, pp. 44905-44 927, 2021.

[9] R. W. Ahmad, K. Salah, R. Jayaraman, I. Yaqoob, and M. Omar, "Blockchain for waste management in smart cities: A survey," 2021.

[10] Y. He, H. Li, X. Cheng, Y. Liu, C. Yang, and L. Sun, "A blockchain based truthful incentive mechanism for distributed P2P applications," IEEE Access, vol. 6, pp. 27 324-27335, 2018.

[11] G. G. Dagher, J. Mohler, M. Milojkovic, and P. B. Marella, "Ancile: Privacy-preserving framework for access control and interoperability of electronic health records using blockchain technology," Sustainable cities and society, vol. 39, pp. 283-297, 2018.

[12] W. Ren, J. Hu, T. Zhu, Y. Ren, and K.-K. R. Choo, "A flexible method to defend against computationally resourceful miners in blockchain Proofof-Work," Information Sciences, vol. 507, pp. 161-171, 2020.

[13] O. Vashchuk and R. Shuwar, "Pros and cons of consensus algorithm Proof-of-Stake: Difference in the network safety in Proof-of-Work and Proof-of-Stake," Electronics and Information Technologies, vol. 9, no. 9, pp. 106-112, 2018.

[14] R. D. Tomlinson, "Let's look to Proof-for-Authority," Childhood Education, vol. 31, no. 3, pp. 137-140, 1954.

[15] M. Niranjanamurthy, B. Nithya, and S. Jagannatha, "Analysis of blockchain technology: Pros, cons and SWOT," Cluster Computing, vol. 22, no. 6, pp. 14 743-14 757, 2019.

[16] I.-C. Lin and T.-C. Liao, "A survey of blockchain security issues and challenges," IJ Network Security, vol. 19, no. 5, pp. 653-659, 2017.
[17] S. S. Sabry, N. M. Kaittan, and I. Majeed, "The road to the blockchain technology: Concept and types," Periodicals of Engineering and Natural Sciences (PEN), vol. 7, no. 4, pp. 1821-1832, 2019.

[18] T. Koens and E. Poll, "What blockchain alternative do you need?" in Data Privacy Management, Cryptocurrencies and Blockchain Technology. Springer, 2018, pp. 113-129.

[19] Yei Sung Kim, "Blockchain Benefits: Driving freight bill audit and pay savings in oil and gas," [Online]. Accessed on 05/05/2021, January 2018, https://www.accenture.com/_acnmedia/PDF-77/Accenture-13584ACN-RES-Blockchain-FBAP-Brochure-2018-final.pdf.

[20] Dmitriy Malets, "13 Benefits Blockchain Offers the Oil and Gas Industry," [Online]. Accessed on 09/05/2021, April 2021, https://www.pipelineoilandgasnews.com/interviewsfeatures/features/2021/ january/blockchain-to-change-how-oil-and-gas-companies-operate/.

[21] A. K. M. B. Haque, M. R. Hasan, and M. Zihad, "SmartOil: Blockchain and smart contract-based oil supply chain management," 042021.

[22] H. Lu, K. Huang, M. Azimi, and L. Guo, "Blockchain technology in the oil and gas industry: A review of applications, opportunities, challenges, and risks," Ieee Access, vol. 7, pp. 41 426-41 444, 2019.

[23] L. A. Ajao, J. Agajo, E. A. Adedokun, and L. Karngong, "Crypto hash algorithm-based blockchain technology for managing decentralized ledger database in oil and gas industry," $J$-Multidisciplinary Scientific Journal, vol. 2, no. 3, pp. 300-325, 2019.

[24] J. Aslam, A. Saleem, N. T. Khan, and Y. B. Kim, "Factors influencing blockchain adoption in supply chain management practices: A study based on the oil industry," Journal of Innovation \& Knowledge, vol. 6, no. 2, pp. 124-134, 2021.

[25] Y. Wang, S. Huang, and X. Yu, "An oil and gas big data sharing model based on blockchain technology," in IOP Conference Series: Earth and Environmental Science, vol. 651, no. 3. IOP Publishing, 2021, p. 032105 .

[26] Á. G. Villacreses Ponce, "Blockchain application for the supply chain of the ecuadorian oil industry," B.S. thesis, Universidad de Investigación de Tecnología Experimental Yachay, 2020.

[27] Y. Miao, J. Song, H. Wang, L. Hu, M. M. Hassan, and M. Chen, "Smart Micro-GaS: A cognitive micro natural gas industrial ecosystem based on mixed blockchain and edge computing," IEEE Internet of Things Journal, 2020.

[28] Etienne Amic, "Re-imagining Post-Trade Management," online, accessed 3/25/2021, March 2018, https://www.vakt.com/.

[29] Thoughts work, "Vakt: Building the world's first enterprise-level blockchain platform," online, accessed 3/25/2021, January 2019, https://www.thoughtworks.com/clients/vakt.

[30] Laure Sadreux, "Natixis, IBM and Trafigura introduce first-ever Blockchain solution for U.S. crude oil market," online, accessed 3/25/2021, March 2017, https://www.natixis.com/natixis/en/natixis-ibmand-trafigura-introduce-first-ever-blockchain-solution-for-u-s-crude-oilmarket-rep_95395.html.

[31] Tyler Smith, "Blockchain Technology in Oil and Gas Industry," online, accessed 3/25/2021, August 2017, https://www.aipn.org/forms/meeting/MeetingFormPublic/.

[32] Infosys Lim, "Oil and Gas industry-blockchain, the disruptive technology," online, accessed 3/25/2021, January 2018, https://www.infosys.com/industries/oil-and-gas/featuresopinions/Documents/blockchain-disruptive-force.pdf.

[33] Mohamed El Masri, "Canadian Windermere Capital to use Blockchain based PermianChain securities platform," online, accessed 3/25/2021, December 2020, https://www.unlock-bc.com/news/202012-27/canadian-windermere-capital-to-use-blockchain-based-permianchain-securities-platform.

[34] World Oil, "PermianChain Technologies has launched blockchain-based O\&G trading, investment platform prototype," online, accessed 3/25/2021, January 2019, https://www.worldoil.com/news/2019/1/21/permianchain-technologieshas-launched-blockchain-based-og-trading-investment-platformprototype.

[35] Y. Tian, Z. Lu, P. Adriaens, R. E. Minchin, A. Caithness, and J. Woo, "Finance infrastructure through blockchain-based tokenization," Frontiers of Engineering Management, vol. 7, no. 4, pp. 485-499, 2020.

[36] Zara Zamani, "ChainTalk," online, accessed 3/28/2021, April 2020, https://www.oilandgas360.com/ziyen-energy-ceo-alastair-caithness-tobe-guest-speaker-on-swedens-leading-blockchain-show-chaintalk/.

[37] Maksym Vasylchykov, "Oil and Gas A Tokenization MiracleSource: Pixabay Oil and Gas, a Tokenization Miracle," online, accessed 3/28/2021, October 2019, https://businessblockchainhq.com/businessblockchain-news/oil-gas-tokenization-miracle/. 
[38] Talal Husseini, "ADNOC and IBM develop blockchain for oil and gas operations," online, accessed 3/28/2021, December 2018, https://www.offshore-technology.com/news/adnoc-blockchain-for-oiland-gas/.

[39] Ledger Insights, "Abu Dhabi Oil reveals IBM blockchain supply chain pilot," online, accessed 3/30/2021, March 2019, https://www.ledgerinsights.com/abu-dhabi-oil-ibm-blockchain/.

[40] Info Sys, "Oil and Gas industry- Blockchain, the disruptive force of the 21 st century (view point)," [Online]. Accessed on 4/05/2021, July 2018, https://infosys.com/.

[41] M. A. Adegboye, W.-K. Fung, and A. Karnik, "Recent advances in pipeline monitoring and oil leakage detection technologies: Principles and approaches," Sensors, vol. 19, no. 11, p. 2548, 2019.

[42] E. Hauge, O. M. Aamo, and J.-M. Godhavn, "Model based pipeline monitoring with leak detection," IFAC Proceedings Volumes, vol. 40, no. 12, pp. 318-323, 2007.

[43] D. Zaman, M. K. Tiwari, A. K. Gupta, and D. Sen, "A review of leakage detection strategies for pressurised pipeline in steady-state," Engineering Failure Analysis, vol. 109, p. 104264, 2020.

[44] Ondiflo F, "Blockchain Use Cases for Midstream Oil \& Gas," [Online]. Accessed on 09/05/2021, September 2018, https://media.consensys.net/blockchain-use-cases-for-midstream-oilgas-609033457e33.

[45] Research Guides, "Oil and Gas Industry: A Research Guide," online, accessed 04/01/2021, March 2019, https://guides.loc.gov/oil-and-gasindustry/midstream/modes/.

[46] W. Lin, N. Zhang, and A. Gu, "LNG (liquefied natural gas): A necessary part in china's future energy infrastructure," Energy, vol. 35, no. 11, pp. 4383-4391, 2010.

[47] P. Mishra, M. Bolic, M. C. Yagoub, and R. F. Stewart, "RFID technology for tracking and tracing explosives and detonators in mining services applications," Journal of Applied Geophysics, vol. 76, pp. 33-43, 2012.

[48] K. Salah, A. Alfalasi, M. Alfalasi, M. Alharmoudi, M. Alzaabi, A. Alzyeodi, and R. Ahmad, "IoT-enabled shipping container with environmental monitoring and location tracking," in IEEE 17th Annual Consumer Communications \& Networking Conference (CCNC). IEEE, 2020, pp. 1-6.

[49] R. K. Perrons, "How innovation and $r \& d$ happen in the upstream oil \& gas industry: Insights from a global survey," Journal of Petroleum Science and Engineering, vol. 124, pp. 301-312, 2014.

[50] JAMES CHEN, "Exploration \& Production (E\&P)," online, accessed 04/04/2021, October 2020, https://www.investopedia.com/terms/e/exploration-productioncompany.asp.

[51] R. K. Perrons and A. Hems, "Cloud computing in the upstream oil \& gas industry: A proposed way forward," Energy Policy, vol. 56, pp. 732-737, 2013.

[52] M. C. Solheim and R. Tveterås, "Benefitting from co-location? evidence from the upstream oil and gas industry," The Extractive Industries and Society, vol. 4, no. 4, pp. 904-914, 2017.

[53] M. Mohammadpoor and F. Torabi, "Big data analytics in oil and gas industry: An emerging trend," Petroleum, 2018.

[54] H. Wiseman, "Trade secrets, disclosure, and dissent in a fracturing energy revolution," Colum. L. Rev. Sidebar, vol. 111, p. 1, 2011.

[55] X.-y. Zhang, J.-n. Wang, Y.-j. Guo, et al., "Advances and trends in logging while drilling technology," Well Logging Technology, vol. 30, no. 1, p. 10, 2006.

[56] J. D. Campbell, A. K. Jardine, and J. McGlynn, Asset management excellence: Optimizing equipment life-cycle decisions. CRC Press, 2016.

[57] Marcus Mares, "ASSET TRACKING FOR THE OIL \& GAS SERVICES IMPROVES PROFITS ," [Online]. Accessed on 19/05/2021, April 2021, https://tgi-connect.com/asset-tracking-for-the-oil-gasservices-improves-profits/.

[58] Worldwide Procurement Service, "Oil \& Gas Industry Equipment Procurement and Supply of Materials and Equipment for Oil and Gas Industries," online, accessed 04/07/2021, October 2020, http://www.worldwideprocurementservices.com/oil-gas-miningprocurement/oil-gas/.

[59] S. Vorarat, A. Al-Hajj, et al., "Developing a model to suit life cycle costing analysis for assets in the oil and gas industry," in SPE Asia Pacific conference on integrated modelling for asset management. Society of Petroleum Engineers, 2004.

[60] E. B. Swanson, "The dimensions of maintenance," in Proceedings of the 2nd international conference on Software engineering, 1976, pp. 492-497.
[61] I. Graham et al., Working conditions of contract workers in the oil and gas industries. ILO, 2010.

[62] Mukund Srivathsa, "Oil and Gas Refining \& Marketing Billing Process And Common Requirements," online, accessed 04/07/2021, October 2020, https://blogs.sap.com/2020/10/09/oil-and-gas-refining-marketingbilling-process-and-common-requirements/.

[63] David Yager, "The Broken Payment Model That Costs The Oil Industry Millions," online, accessed 04/07/2021, July 2015, https://oilprice.com/Energy/Energy-General/The-Broken-PaymentModel-That-Costs-The-Oil-Industry-Millions.html.

[64] A. Asgaonkar and B. Krishnamachari, "Solving the buyer and seller's dilemma: A dual-deposit escrow smart contract for provably cheat-proof delivery and payment for a digital good without a trusted mediator," in 2019 IEEE International Conference on Blockchain and Cryptocurrency (ICBC). IEEE, 2019, pp. 262-267.

[65] R. W. Ahmad, H. Hasan, R. Jayaraman, K. Salah, and M. Omar, "Blockchain applications and architectures for port operations and logistics management," Research in Transportation Business \& Management, p. $100620,2021$.

[66] Intertek, "Environmental Compliance for the Oil and Gas Industry," online, accessed 04/07/2021, October 2020, https://www.intertek.com/exploration-production/support/oil-gasenvironmental-compliance/.

[67] N. Tiitmamer, "Compliance with petroleum industry transparency and accountability rules in south sudan," 2019.

[68] Kathryn Mutz, "Compliance and Enforcement," online, accessed 04/09/2021, January 2015, http://www.oilandgasbmps.org/resources/compliance_enforcement.php.

[69] A. L. Mitchell and E. A. Casman, "Economic incentives and regulatory framework for shale gas well site reclamation in pennsylvania," Environmental science \& technology, vol. 45, no. 22, pp. 9506-9514, 2011.

[70] M. Khalifeh and A. Saasen, Introduction to Permanent Plug and Abandonment of Wells. Springer Nature, 2020.

[71] Z. Mingaleva, N. Vukovic, I. Volkova, and T. Salimova, "Waste management in green and smart cities: A case study of Russia," Sustainability, vol. 12 , no. 1, p. 94, 2020.

[72] S. Saberi, M. Kouhizadeh, J. Sarkis, and L. Shen, "Blockchain technology and its relationships to sustainable supply chain management," International Journal of Production Research, vol. 57, no. 7, pp. 21172135, 2019.

[73] Y. Chen, H. Li, K. Li, and J. Zhang, "An improved P2P file system scheme based on IPFS and blockchain," in IEEE International Conference on Big Data (Big Data). IEEE, 2017, pp. 2652-2657.

[74] T. Khalid, M. A. K. Abbasi, M. Zuraiz, A. N. Khan, M. Ali, R. W. Ahmad, J. J. Rodrigues, and M. Aslam, "A survey on privacy and access control schemes in Fog computing," International Journal of Communication Systems, vol. 34, no. 2, p. e4181, 2021.

[75] T. T. Huynh, T. D. Nguyen, and H. Tan, "A survey on security and privacy issues of blockchain technology," in 2019 International Conference on System Science and Engineering (ICSSE). IEEE, 2019, pp. $362-367$.

[76] C. Meijer, "Remaining challenges of blockchain adoption and possible solutions," 2020.

[77] C. Cachin et al., "Architecture of the hyperledger blockchain fabric," in Workshop on distributed cryptocurrencies and consensus ledgers, vol. 310, no. 4. Chicago, IL, 2016.

[78] P. Praitheeshan, L. Pan, and R. Doss, "Private and trustworthy distributed lending model using hyperledger besu," SN Computer Science, vol. 2, no. 2, pp. 1-19, 2021.

[79] T. Espel, L. Katz, and G. Robin, "Proposal for protocol on a quorum blockchain with zero knowledge." IACR Cryptol. ePrint Arch., vol. 2017, p. 1093, 2017.

[80] J. Partala, T. H. Nguyen, and S. Pirttikangas, "Non-interactive zeroknowledge for blockchain: A survey," IEEE Access, vol. 8, pp. 227945 227 961, 2020.

[81] J. Bhosale and S. Mavale, "Volatility of select crypto-currencies: A comparison of bitcoin ethereum and litecoin," Annu. Res. J. SCMS, Pune, vol. 6, 2018.

[82] G. Wang, Z. J. Shi, M. Nixon, and S. Han, "Sok: Sharding on blockchain," in Proceedings of the 1st ACM Conference on Advances in Financial Technologies, 2019, pp. 41-61.

[83] S. Schulte, M. Sigwart, P. Frauenthaler, and M. Borkowski, "Towards blockchain interoperability," in International Conference on Business Process Management. Springer, 2019, pp. 3-10.

[84] C. R.W, "Blockchain and interoperability: Key to mass adoption," 2020. 
[85] K. Salah, M. H. U. Rehman, N. Nizamuddin, and A. Al-Fuqaha, "Blockchain for AI: Review and open research challenges," IEEE Access, vol. 7, pp. $10127-10149$, (2019).

[86] M. Wohrer and U. Zdun, "Smart contracts: Security patterns in the Ethereum ecosystem and Solidity," in 2018 International Workshop on Blockchain Oriented Software Engineering (IWBOSE). IEEE, 2018, pp. 2-8.

[87] S. Rouhani and R. Deters, "Security, performance, and applications of smart contracts: A systematic survey," IEEE Access, vol. 7, pp. 50759 $50779,2019$.

[88] P. Praitheeshan, L. Pan, J. Yu, J. Liu, and R. Doss, "Security analysis methods on Ethereum smart contract vulnerabilities: A survey," arXiv preprint arXiv:1908.08605, 2019.

[89] L. Marchesi, M. Marchesi, G. Destefanis, G. Barabino, and D. Tigano, "Design patterns for gas optimization in Ethereum," in 2020 IEEE International Workshop on Blockchain Oriented Software Engineering (IWBOSE). IEEE, 2020, pp. 9-15.

[90] T. Chen, X. Li, Y. Wang, J. Chen, Z. Li, X. Luo, M. H. Au, and X. Zhang, "An adaptive gas cost mechanism for Ethereum to defend against under-priced DOS attacks," in International Conference on Information Security Practice and Experience. Springer, 2017, pp. 3-24.

[91] X. Liu, K. Muhammad, J. Lloret, Y.-W. Chen, and S.-M. Yuan, "Elastic and cost-effective data carrier architecture for smart contract in blockchain," Future Generation Computer Systems, vol. 100, pp. 590599, 2019. 